\title{
Editorial
}

\section{Discharge Criteria in Ambulatory Surgery}

Day care surgery is also called as outpatient surgery, ambulatory surgery, fast track surgery, and same-day surgery. An anesthesiologist Ralph Waters built, opened, and managed the first independent outpatient surgery, and outpatient anesthesia clinic in Sioux City, Iowa in the early 1900, to provide surgical care to patients whose operations were thought to be highly demanding for a surgeon's office yet did not require overnight hospitalization. The advances in surgery, anesthesia, and pain management have allowed the use of this modality of care with a consequent reduction in the need for hospitalization and the cost. The incidence of day surgeries in the United States is $80 \%$ of total surgeries and expected it to be $>75 \%$ of total surgeries in the UK. Even though data are not available for India, there

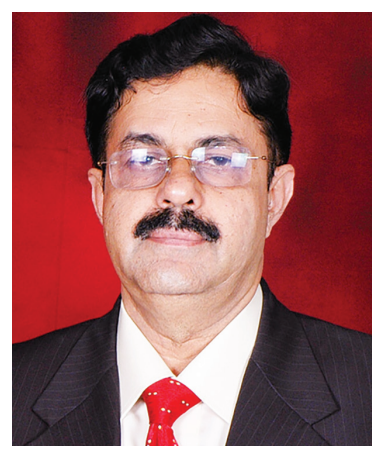
is huge potential in view of a massive population of over 3 billion, and recent expansion of hospitals in the private sector has created an opportunity for increase in day surgery in India. ${ }^{1-3}$

Association of Anesthetists of Great Britain and Ireland British Association of Day Surgery in their Summary on day case and short stay surgery quote ${ }^{2}$ :

- Day surgery is a continually evolving specialty performed in a range of ways across different units.

- At present the indications for complex surgical procedures on day care basis have increased with a wider range of patients now considered suitable.

- Careful preoperative preparation and nurse-led discharge using set protocols are fundamental to safe and effective day surgery units.

- Fitness for a procedure should relate to the patient's health as determined at preoperative preparation and not limited by arbitrary limits, such as American society of anesthesiologists (ASA) status, age, or body mass index.

- Patients presenting with acute conditions requiring urgent surgery can be efficiently and effectively treated as day cases via a semi-elective pathway.

- Regional anesthetic techniques, including central neuraxial blockade and a range of brachial plexus and paravertebral blocks, may be used effectively for day surgery.

\section{DEFINITIONS OF DAY CARE/AMBULATORY SURGERY}

- A surgical day care is defined by the Royal College of Surgeons in Ireland and England ${ }^{2}$ as "a patient who is admitted for investigation or operation on a planned non-resident basis and who also requires facilities for recovery."

\section{RECOVERY AFTER AMBULATORY SURGERY}

After surgery, recovery is a continuous process that begins when the patient recovers from anesthesia and stops when the patient has returned to preoperative physiological and functional state.

Three stages of recovery after ambulatory surgery are:

1. Early $\rightarrow$ time interval during which patients emerge from anesthesia, recover control of their protective reflexes, and resume early motor activity - Aldrete score - operating room.

2. Intermediate $\rightarrow$ recovery room - begin to ambulate, drink fluids, void, and prepare for discharge.

3. Late $\rightarrow$ period starts when the patient is discharged home and continues until complete functional recovery is achieved and the patient is able to resume normal activities of daily living.

\section{IDEAL RECOVERY AREA}

- Should be well equipped and staffed to deal with common postoperative nausea and vomiting (pain) problems as well as emergencies (hemorrhages, cardiovascular events).

- The anesthetist and surgeons must be contactable as soon as possible to help deal with problems and effectively treat them.

- Patients and their caregivers or attenders should be provided with written information that includes warning signs of possible complications and where to seek, and from whom to seek help. 


\section{DISCHARGE CRITERIA AFTER AMBULATORY SURGERY}

- Must be awake, alert, and orientated to person, place, and time.

- Should have stable vital signs.

- If permitted, patients should be able to tolerate oral fluids.

- Pain should be manageable with oral analgesics.

- There must be minimal nausea, vomiting, and dizziness.

- There must be no or minimal bleeding.

- Upon discharge patients must be given:

- Verbal and written instructions

- A discharge prescription

- Relevant contact numbers for emergencies

- Patients must be informed that they will be contacted the next day by the day care staff to enquire about their well-being.

Postoperative discharge is usually based on the following three phases of Recovery. ${ }^{4}$

- Phase 1: Ready to go toward recovery - patient is awake, pain is controlled. Aldrete score is useful here.

- Phase 2: Phase of home readiness - we use postanesthesia discharge scoring system.

- Phase 3: Phase of late recovery - patient's full physiological and psychological recovery occurs at home, which may take several weeks or months.

We make use of a scoring system to assess the suitability for discharge. When the patients attain a score of 9 or more and have a responsible adult person to escort, are considered fit for discharge. Discharge after a central neuraxial block is done when the patient regains normal sensation, muscle strength, and proprioceptive sense. Sympathetic nervous system function must also be restored before discharge.

\section{POSTANESTHETIC RECOVERY SCORING SYSTEM - PAS - (MODIFIED ALDRETE) ${ }^{4}$}

\section{Consciousness}

- A point score of $2 \rightarrow$ patient is fully awake, able to answer questions, and call for assistance. In cases of patients with altered level of consciousness before surgery, score of 2 is allotted when the patient reaches preoperative conscious level.

- A point score of $1 \rightarrow$ patient is drowsy but responds easily to verbal commands.

- A point score of $0 \rightarrow$ no response is elicited to verbal commands. It is better to avoid painful stimulation to elicit a response.

- The minimal score of 1 must be achieved in this category before discharge.

\section{Respiration}

- A point score of $2 \rightarrow$ patient is able to breathe deeply and cough.

- A point score of $1 \rightarrow$ patient exhibits signs of dyspnea or has difficulty breathing and clearing secretions or requires supportive measures to maintain airway patency.

- A score of $0 \rightarrow$ patient is apneic or requires assisted ventilation.

- A score of 2 must be achieved and maintained in this category for a minimum of $1 \frac{1}{2}$ hours before discharge.

\section{Circulation: Blood Pressure}

- Score of $2 \rightarrow$ blood pressure (systolic/MAP) reading is $\pm 20 \mathrm{~mm} \mathrm{Hg}$ of the preanesthetic level.

- Score of $1 \rightarrow$ blood pressure (systolic/MAP) reading is $> \pm 20-35 \mathrm{~mm} \mathrm{Hg}$ of the preanesthetic level.

- Score of $0 \rightarrow$ blood pressure (systolic/MAP) reading is $> \pm 35-50 \mathrm{~mm} \mathrm{Hg}$ of the preanesthetic level.

- If systolic blood pressure $<90$, or $>200 \mathrm{~mm} \mathrm{Hg}$, or exhibiting a blood pressure other than their baseline preanesthetic blood pressure then it must be evaluated by an anesthesiologist prior to discharge.

- Before discharge, patients should attain a score of least 1 and must be maintained for at least three consecutive readings at 15 minutes intervals. The above-defined parameters in paragraph " $\mathrm{d}$ " must also be attained.

\section{Circulation: Heart Rate}

- A score of $2 \rightarrow$ heart rate is \pm 20 beats/minute of the preanesthetic level.

- A score of $1 \rightarrow$ heart rate is $\pm 20-35$ beats/minute of the preanesthetic level. 
- A score of $0 \rightarrow$ heart rate is $\pm 35-50$ beats/minute of the preanesthetic level.

- Patients with a heart rate of $<50$ beats/minute, $>110$, or exhibiting a cardiac rhythm other than their baseline preanesthetic rhythm must be evaluated by an anesthesiologist prior to discharge.

- Before discharge, patients should attain a score of least 1 or higher than 1 and must also achieve for three consecutive measurements at 15 minutes intervals. The above-defined parameters in paragraph " $\mathrm{d}$ " must also be attained.

\section{Oxygen Saturation}

- A score of $2 \rightarrow \mathrm{SpO}_{2}$ meets or exceeds the anesthesiologist's parameters on room air.

- A score of $1 \rightarrow \mathrm{SpO}_{2}$ meets or exceeds the anesthesiologist's parameters on supplemental $\mathrm{O}_{2}$.

- A score of $0 \rightarrow$ minimum $\mathrm{SpO}_{2}$ level as established by the anesthesiologist cannot be maintained.

- Patient should attain a score of 1 or more than 1 , before the discharge.

\section{Activity}

- A score of $2 \rightarrow$ patient is able to move all four extremities on command, or motor activity has returned to the patient's preoperative status (if a deficit exists).

- A score of $1 \rightarrow$ patient is able to move only three extremities.

- A score of $0 \rightarrow$ patient is able to move only two extremities.

- Patient must achieve a score of 2 before discharge. It is also subject to anesthesiologist's decision.

- Patients who receive a regional nerve block, a score of 3 is desirable which must be certified by the anesthesiologist before discharge.

Pain Scale: Document the pain scale using the appropriate scoring scale for the patient.

\section{POSTANESTHESIA DISCHARGE SCORING (PADS) SYSTEM ${ }^{4}$}

- Vital signs

- Within $20 \%$ of the preoperative value

- $1-20$ to $40 \%$ of the preoperative value

- $0-40 \%$ of the preoperative value

- Ambulation

- 2 - Steady gait/no dizziness

- 1 - With assistance

- 0 - No ambulation/dizziness

- Nausea and vomiting

- 2 - Minimal

- 1 - Moderate

- 0 -Severe

- Pain

- 2 - Minimal

- 1 - Moderate

- 0 -Severe

- Surgical bleeding

- 2 - Minimal

- 1 - Moderate

- 0 -Severe

Document the pain scale, using the appropriate scoring scale for the patient.

\section{DISCHARGE OF OUTPATIENTS FROM PHASES I AND II POSTANESTHESIA CARE}

- Outpatients discharged to the phase II recovery area will meet the above discharge criteria and also include the following:

- Patient is oriented to preanesthetic level and the patient may be awake and alert.

- Patient tolerates sitting in an upright position without signs or symptoms of orthostatic hypotension. 
- Patients who received (spinal/epidural) anesthesia should regain sensory and motor function of the lower extremities and demonstrate the ability to stand and walk with minimal assistance.

- If patients have been administered upper limb regional blocks like brachial plexus blocks, they must get an arm sling before they regain full sensory and motor control.

- If patients have been administered femoral, popliteal, sciatic, or ankle block, they must get crutches and should be instructed not to bear the weight until full sensory and motor functions have returned.

- If patients are discharged home directly from phase I, they must meet the set criteria as mentioned earlier.

\section{DISCHARGE OF PATIENTS BY CRITERIA IS A STANDARDIZED PROCEDURE}

However, the following simple recovery criteria are useful in day-to-day clinical practice ${ }^{1}$ :

- Simple psychomotor tests like memory and sensory motor coordination tests.

- With spinal anaesthesia, it is generally accepted that motor and sensory functions return before recovery of sympathetic nerve system.

- Two successive orthostatic mean arterial blood pressure (MAP) decreases of $10 \%$ or less.

- Prior to ambulation, patients should have normal perianal sensation which refers to S4-S5 dermatome, should be able to plantarflex the foot, and regain sense of proprioception of the big toe.

\section{FAST TRACK DISCHARGE CRITERIA ${ }^{5}$}

Recently, they have developed criteria utilizing the mnemonic "SPEEDS" (saturation, pain, extremity movement, emesis, dialogue, stable vitals signs) to assess and decide which patients would not require phase I nursing intervention and could do transition to phase II recovery. Instead of using a point system, the SPEEDS criteria require a simple "yes" or "no" response to each parameter. A patient must have a response of "yes" to all parameters to progress to phase II recovery. ${ }^{5}$

The following discharge criteria are used in fast tracking some of the patients eligible for discharge.

\begin{tabular}{ll}
\hline Speeds criteria & Score \\
\hline $\begin{array}{l}\text { Oxygen } \\
\text { Saturation }>90 \%\end{array}$ & $\mathrm{Y} / \mathrm{N}$ \\
$\begin{array}{l}\text { Room air } \\
\text { Pain control } \\
\text { (VAS }<4)\end{array}$ & \\
$\begin{array}{l}\text { Extremity } \\
\text { Movement } \\
\text { (moves all four) }\end{array}$ & $\mathrm{Y} / \mathrm{N}$ \\
$\begin{array}{l}\text { Emesis control } \\
\text { Dialogue } \\
\text { (oriented person/place) }\end{array}$ & \\
$\begin{array}{l}\text { Stable vital signs } \\
\text { SBP }>90 \text { and }<180\end{array}$ & $\mathrm{Y} / \mathrm{N}$ \\
$\mathrm{HR}>50$ and $<110$ & $\mathrm{Y} / \mathrm{N}$ \\
\hline
\end{tabular}

\section{DISCHARGE INSTRUCTIONS}

The following are to be given to patients at discharge. Nurse should explain instructions to patient.

- You should be under the care of a responsible adult for 24 hours after your discharge.

- For the next 24 hours:

- Do not drive any vehicle.

- Do not use power tools/appliances which may cause harm or injury.

- Patients should not make any important decisions or sign any legal documents for 24 hours.

- Do not consume alcohol.

- Nausea and vomiting are common after general anesthesia. If you have these take the medication provided for this. If it persists, you must call the contact numbers provided for advice. 
Call the telephone numbers provided, if you have any of the following:

- Difficulty in passing urine

- Fever

- Bleeding

- Redness or infection at the site of the operation

- Any other complication that you are worried about Surgeons should give surgical discharge instructions to be given separately.

\section{CONTROVERSIES IN THE DISCHARGE CRITERIA}

Voiding prior to discharging the patients: Sympathetic nerve supply to bladder and urethra may be blocked and hence cause retention of urine. The subarachnoid block has to regress beyond S-3 level for the return of micturation reflex. Hence, in patients with low risk of urinary retention is predicted, voiding before discharge may not be necessary.

- Is voiding necessary before discharge?

- Voiding is not a requirement before discharge

- Risks

- Ultrasonography.

- Is an escort needed following ambulatory surgery?

- As per the American Society of Anesthesiologists (ASA) recommendations, it is a must.

- After ambulatory surgery when can you drive?

- Recently, a prospective study involving ambulatory surgery has shown that patients have lower alertness levels and impaired driving skills preoperatively and 2 hours postoperatively. Alertness and driving skills returned to normal levels at 24 hours.

Many would ask whether to allow ambulatory patients to drink or withhold in the early postoperative period: Re-intake of oral fluids and spontaneous voiding are no longer required for discharge after outpatient surgery. Mandatory drinking may in fact provoke nausea and vomiting.

- Is oral fluid intake necessary before discharge?

- If forced, higher incidence of vomiting and a prolonged hospital stay in children has been reported.

- In adults, drinking did not influence the incidence of postoperative nausea and vomiting or length of hospital stay.

- Oral fluids intake is not a requirement prior to discharge.

Recovery and discharge include:

- Phase of early recovery - awakening and recovery of vital reflexes. It takes lace in the recovery area.

- Phase of intermediate recovery - the patient has recovered to a point of "home readiness" so that he could be sent home.

- Phase of late recovery - the patient has recovered to the point of "street fitness," after which he resumes his normal life.

Normally patients are discharged from the day unit when they have completed the intermediate phase of recovery.

The above various criteria can also be classified as:

- Essential invariable criteria

- Stable vital signs

- Orientated to preoperative stage

- Minimal nausea and vomiting

- Controllable pain

- No significant bleeding having regard to the procedure

- Variable criteria

- Micturition prior to discharge is essential following epidural or spinal anesthesia. This may also be essential following certain day care surgical procedures.

- In certain cases patients may have to stay for a fixed length of stay after surgery. 
- This may not be a common requirement after all surgical procedures.

- May be deemed necessary after certain procedures to minimize the risk of reactionary hemorrhage at home, e.g., tonsillectomy, thyroidectomy.

- It is more applicable for certain specific procedures.

- The following is disputed criterion:

- Patient's ability to drink oral fluids and retain, before discharge, is increasingly disputed. Oral fluids may provoke nausea and vomiting and cause distress to patients.

The following criteria which are considered nonmedical should preferably be met before discharge:

- The patients should be accompanied by responsible adults after discharge and to be with them at home at least for the first 24 hours following surgery.

- Access to a functioning telephone at home.

\section{POSTOPERATIVE INSTRUCTIONS}

- Patient should be given:

- Printed postoperative instructions

- Printed information on whom to contact in case of emergency

- Prescription of necessary drugs

- Adequate and clear follow-up instructions

- Discharge summary of the treatment they have received at the day care unit.

- Who should assess fitness of the patient for discharge from the day care surgery unit?

- It could be either surgeon or anesthesiologist.

- In-charge senior nurse may also be allowed against a hospital approved printed protocol. In cases of any doubt or when there is a problem, either surgeon or anesthesiologist should assess.

\section{CLINICAL AUDIT}

It is worthwhile in initiating clinical audit of these policies and procedures. Quality of care and efficiency are the main components of the audit of day care surgery services. It should also include patients' satisfaction of the day care experience. We should create a database which will be helpful. This database may fail to effect change unless the information is spread to the day care surgery clients. Monthly graphs and figures detailing all outcomes and trends should be informed to everyone involved, particularly to key individuals empowered to influence change. ${ }^{2}$

\section{SUMMARY}

- Prior to discharge from the day case unit:

- Patients should have stable vital signs.

- Patients should be alert and orientated.

- Patients should be comfortable and without pain.

- Patients should be able to walk.

- Patients should be able to tolerate oral fluids with or without minimal nausea and vomiting.

- Patients should be given follow-up instructions and discharge summary.

- They should be provided with contact telephone numbers in case of any emergency.

\section{CONCLUSION}

Day surgery is a process of care by which suitable patients are managed with admission, treatment, and discharge on the same calendar day, ideally within a dedicated and designated environment.

- Patients undergoing day care surgery move through a quantum of medical care to which physicians and surgeons contribute to ensure the best outcome possible.

- The anesthetic techniques that use short-acting drugs and minimize postoperative mortality and morbidity should become the focus in day surgery anesthesia.

- We should follow certain policies to manage postoperative pain, nausea, vomiting, voiding, and discharge from hospital which will in turn result in good outcome measures. 


\section{REFERENCES}

1. Harsoor SS. Changing concepts in anaesthesia for day case surgery. Indian J Anaesth 2010 Nov-Dec;54(6):485-488.

2. Verma R, Alladi R, Jackson I, Johnston I, Kumar, Page R, Smith I, Stocker M, Tickner C, Williams S, et al. Guide lines - day case and short stay surgery: 2. Anaesthesia 2011 May;66(5):417-434.

3. Row TN. Progress of day surgery in India. Ambul Surg 2010 Apr:16:15-16.

4. Gangadhar SB, Gopal TM, S, Paramesh KS. Rapid emergence of day-care anaesthesia: a review. Indian J Anaesth 2012 Jul-Aug;56(4):336-341.

5. Burke B, Kyker M. Speeds criteria vs modified aldrete and fast-track criteria for evaluating recovery in outpatients. Open J Anesth 2013 Sep;3(7):309-314.

H Sahajananda

Professor and Head

Department of Critical Care and Anesthesiology RajaRajeswari Medical College and Hospital

Bengaluru, Karnataka, India

Phone: +919448085401, e-mail: sahaj_anand@hotmail.com 